\title{
The BinaxNOW pneumococcal antigen test: An adjunct for diagnosis of pneumococcal bacteraemia
}

\begin{tabular}{|c|c|}
\hline \multicolumn{2}{|c|}{$\begin{array}{l}\text { Authors: } \\
\text { Hafsah D. Tootla } \\
\text { Colleen Bamford } \\
\text { Chad M. Centner }^{1,2} \\
\text { Clinton Moodley }^{1,2}\end{array}$} \\
\hline \multicolumn{2}{|c|}{$\begin{array}{l}\text { Affiliations: } \\
{ }^{1} \text { Division of Medical } \\
\text { Microbiology, Faculty of } \\
\text { Health Sciences, University } \\
\text { of Cape Town, Cape Town, } \\
\text { South Africa }\end{array}$} \\
\hline \multicolumn{2}{|c|}{$\begin{array}{l}{ }^{2} \text { National Health Laboratory } \\
\text { Service, Microbiology, Groote } \\
\text { Schuur Hospital, Cape Town, } \\
\text { South Africa }\end{array}$} \\
\hline \multicolumn{2}{|c|}{$\begin{array}{l}\text { Corresponding author: } \\
\text { Hafsah Tootla, } \\
\text { hafsah.tootla@gmail.com }\end{array}$} \\
\hline \multicolumn{2}{|c|}{$\begin{array}{l}\text { Dates: } \\
\text { Received: } 04 \text { Sept. } 2020 \\
\text { Accepted: } 05 \text { Jan. } 2021 \\
\text { Published: } 15 \text { Apr. } 2021\end{array}$} \\
\hline \multicolumn{2}{|c|}{$\begin{array}{l}\text { How to cite this article: } \\
\text { Tootla HD, Bamford C, } \\
\text { Centner CM, Moodley C. The } \\
\text { BinaxNOW pneumococcal } \\
\text { antigen test: An adjunct for } \\
\text { diagnosis of pneumococcal } \\
\text { bacteraemia. S Afr J Infect } \\
\text { Dis. 2021;36(1), a244. } \\
\text { https://doi.org/10.4102/ } \\
\text { sajid.v36i1.244 }\end{array}$} \\
\hline \multicolumn{2}{|c|}{$\begin{array}{l}\text { Copyright: } \\
\text { (C) 2021. The Authors. } \\
\text { Licensee: AOSIS. This w } \\
\text { is licensed under the } \\
\text { Creative Commons } \\
\text { Attribution License. }\end{array}$} \\
\hline \multicolumn{2}{|l|}{ Read online: } \\
\hline 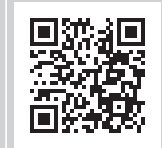 & $\begin{array}{l}\text { Scan this QR } \\
\text { code with your } \\
\text { smart phone or } \\
\text { mobile device } \\
\text { to read online. }\end{array}$ \\
\hline
\end{tabular}

Background: Culture remains the diagnostic standard for Streptococcus pneumoniae bacteraemia but is limited by time to identification, prior antibiotics and bacterial autolysis. Cultureindependent methods for detecting S. pneumoniae include PCR and antigen tests. We evaluated an antigen test on blood culture broth for the rapid detection of S. pneumoniae bacteraemia.

Method: We collected 212 signal-positive blood cultures, with gram-positive cocci in pairs, chains or with uncertain morphology. The BinaxNOW S. pneumoniae urinary antigen test, Gram stain, culture and lytA PCR were performed on all samples. Diagnostic accuracy of the antigen test and Gram stain with gram-positive cocci in pairs were compared with culture, polymerase chain reaction (PCR) and the composite of culture and PCR.

Results: Streptococcus pneumoniae was isolated in $26 \%$ of samples, $66 \%$ cultured other grampositive organisms and $8 \%$ of samples had no growth. Sensitivity and negative predictive values of the antigen test were $100 \%$, specificity and positive predictive values were $87 \%-88 \%$ and $76 \%-81 \%$, but increased to $93 \%-96 \%$ and $96 \%-98 \%$ when applied to subsets with grampositive cocci in pairs, or history compatible with respiratory illness or meningitis. Sensitivity $(69 \%-75 \%)$ and specificity (81\%) of Gram stain (gram-positive cocci in pairs) were lower than the antigen test even when applied to the same subsets.

Conclusion: Accurate and rapid diagnosis of S. pneumoniae bacteraemia is challenging. Specificity of this antigen test is limited by cross-reactivity with other gram-positive organisms, but could be improved if Gram stain morphology and clinical history are available. The antigen test is a useful adjunct for rapid diagnosis of S. pneumoniae bacteraemia.

Keywords: Streptococcus pneumoniae; bacteraemia; BinaxNOW; antigen; pneumococcal diagnosis; test.

\section{Introduction}

Invasive pneumococcal disease (IPD) remains a cause of significant morbidity and mortality worldwide despite the introduction of pneumococcal conjugate vaccination programmes. ${ }^{1,2,3}$

The diagnosis of IPD from sterile sites traditionally relies on microscopy indicating gram-positive cocci in pairs and culture of the organism. ${ }^{4}$ Identification of Streptococcus pneumoniae from culture is confirmed by phenotypic methods, such as susceptibility to optochin (ethylhydrocupreine) and bile solubility. Streptococcus pneumoniae is a fastidious organism, easily inhibited by prior antibiotic exposure and prone to autolysis under laboratory conditions.

Culture is also slow, taking $24-48 \mathrm{~h}$ for definitive identification. ${ }^{4,5,6}$ Fortunately, high-level penicillin resistance, defined as minimum inhibitory concentration (MIC) $\geq 2 \mu \mathrm{g} / \mathrm{mL}$, is still relatively rare $(\sim 7 \%)$ in our setting. ${ }^{1}$ Rapid testing would allow for rapid de-escalation of empiric therapy in non-meningitis infections, reducing the selection of resistance.

Alternative culture-independent methods for the diagnosis of IPD include PCR and antigen tests. Polymerase chain reaction (PCR) is more sensitive, less influenced by prior antibiotic exposure, and rapid, but is limited by cost, accessibility and variable diagnostic accuracy depending on the gene target used. Most of the currently used gene targets ( $p l y, l y t A, p s a A$, sodA and $p b p$ ) demonstrated reduced specificity, primarily because of cross-reactivity with other closely related species. ${ }^{4,7,8,9}$

The BinaxNOW S. pneumoniae Antigen Card test (Alere) is a lateral flow immunochromatographic test (ICT), which detects C-polysaccharide cell wall protein, common to all S. pneumoniae serotypes. ${ }^{6,8,10,11}$ It has been validated for use on urine to assist with the diagnosis of 
community-acquired pneumonia (CAP), although crossreactivity with other streptococcal species and S. pneumoniae colonisation in the upper respiratory tract have limited its utility. The test can also be used on specimens from sterile sites such as cerebrospinal fluid for the rapid diagnosis of pneumococcal meningitis. A few studies have explored its use on incubated signal-positive blood cultures, demonstrating its potential for rapid identification of S. pneumoniae bacteraemia, especially when culture does not yield growth of an organism. However, differences in reference methods and inclusion criteria have hindered the comparisons of diagnostic accuracy.5,6,12,13

We compared the BinaxNOW S. pneumoniae Antigen Card ICT with a rigorous reference standard, incorporating both culture and lyt $A \mathrm{PCR}$, to determine if this test could rapidly identify $S$. pneumoniae from selected signal-positive blood cultures.

\section{Methods \\ Study setting and design}

A prospective study was carried out at the National Health Laboratory Service, Groote Schuur Microbiology Laboratory, Cape Town, South Africa, between 01 June 2017 and 31 March 2018. The laboratory provides diagnostic microbiology services to $\sim 1.85$ million people from predominantly low socio-economic backgrounds. We receive approximately 40000 blood cultures annually and use the BacT/ALERT automated blood culture system (BioMérieux, Marcy-l'Etoile, France) for routine testing.

A convenience sampling method was employed, where nonconsecutive signal-positive blood culture bottles with microscopy suggestive of $S$. pneumoniae, namely, grampositive cocci in pairs or chains, or with gram positive cocci in uncertain morphological arrangement, were selected by a single researcher. Samples were only collected during periods of researcher availability in the laboratory. The presence of brown-coloured broth suggestive of autolysis was subjectively noted. Antigen testing was performed within 6 h of Gram staining. Deoxyribonucleic acid extraction and PCR were batched and performed on all samples by the single researcher who was blinded to the results of the other diagnostic tests and clinical presentation, until all tests were completed. Basic clinical and epidemiological information were collected by the attending microbiology trainee through telephonic consultation with the treating doctor and notes were made from the clinical liaison.

\section{Culture}

Blood culture broth from signal-positive bottles was aspirated and Gram staining was performed.

Samples exhibiting gram-positive cocci in pairs, chains or gram-positive cocci of uncertain morphological arrangement were inoculated onto 2\% horse blood agar and 5\% sheep blood agar, the latter with a $5 \mu \mathrm{g}$ optochin disc placed in the main inoculating zone, as per routine laboratory standard operating procedures (SOPs). Both plates were incubated in $5 \% \mathrm{CO}_{2}$ at $37^{\circ} \mathrm{C}$ and inspected after 24 and $48 \mathrm{~h}$. Streptococcus pneumoniae was identified by the presence of alphahaemolytic streptococci with a zone of inhibition around the optochin disk $\geq 14 \mathrm{~mm}$ diameter. ${ }^{14}$ Alpha-haemolytic streptococci with zones of inhibition $<14 \mathrm{~mm}$ diameter were further identified using the Vitek 2 GP-ID card (BioMérieux, Marcy-l'Etoile, France), according to the manufacturer's instructions.

\section{BinaxNOW Streptococcus pneumoniae antigen card test}

Swabs provided by the manufacturer were submerged into $1 \mathrm{~mL}$ aliquots of blood culture broth and inserted into the test card. Test reagent was added and the card sealed. Results were read after $15 \mathrm{~min}$ and interpreted according to the package insert. ${ }^{15}$ Faint reactive lines were recorded as weak positive results.

\section{Deoxyribonucleic acid extraction}

Deoxyribonucleic acid extraction of blood culture broth was performed using the QIAsymphony SP automated extraction instrument with the QIAsymphony Virus/Bacteria mini kit, according to the manufacturer's instructions (Qiagen, Hilden, Germany). Deoxyribonucleic acid extracted from $200 \mu \mathrm{L}$ of blood culture broth was eluted into $60 \mu \mathrm{L}$ of elution buffer and stored at $-70^{\circ} \mathrm{C}$.

\section{Real-time lytA polymerase chain reaction}

Polymerase chain reaction assays were batched and performed using the Rotor-Gene 6000 (Corbett Research) analyser. Each $25 \mu \mathrm{L}$ reaction contained $5 \mu \mathrm{L}$ of extracted DNA (at a 1:10 dilution to reduce the effect of co-purified inhibitors from blood culture broth), $200 \mathrm{nM}$ each of the forward and reverse primer and probe (Table 1-A1), ${ }^{16} 12.5$ $\mu \mathrm{L}$ of LightCycler ${ }^{\circledR} 480$ Probes Master (Roche Life Science) master mix and $6 \mu \mathrm{L}$ of PCR grade water. A no template control and $S$. pneumoniae positive DNA control (S. pneumoniae ATCC 49619) were included in every run. Deoxyribonucleic acid was amplified using the following cycling conditions: $95^{\circ} \mathrm{C}$ for $5 \mathrm{~min}$, then 40 cycles at $95^{\circ} \mathrm{C}$ for $10 \mathrm{~s}$ and $60^{\circ} \mathrm{C}$ for $1 \mathrm{~min}$ with fluorescence detection.

Results were analysed using the Rotor-Gene 6000 Series Software 1.7. Both ICT and PCR were repeated on all discordant samples, with no changes to the initial results noted.

A cycle threshold (CT) cut-off value of $\leq 22$ was used to determinePCR positivity. This cut-off value was experimentally determined by receiver operating characteristic (ROC) curve analysis (Figure 1-A1) and inspection of CT-value distributions of signal-positive samples (Figure 2-A1). For ROC curve 
analysis we considered S. pneumoniae culture-positive samples as true positives, and those with organisms other than $S$. pneumoniae as true negatives. Receiver operating characteristic curve analysis suggested that the optimal CT cut-off value for determining a positive PCR result was between 17 and 22 (area under the curve [AUC] 0.99) and the upper value of $\mathrm{CT} \leq 22$ was selected.

\section{Sample size}

Using the following parameters - prevalence of S. pneumoniae from blood cultures in our laboratory with gram-positive cocci in pairs/chains or cocci with uncertain morphological arrangement $=20 \%$, hypothesised sensitivity and specificity of ICT using signal-positive blood culture broth $=99 \%$ and $82 \%$, respectively, ${ }^{5,6,12,13}$ desired precision $=95 \%$ and desired confidence interval $(\mathrm{CI})=90 \%$ - we estimated the required sample size to be $\geq 201 .^{17,18,19}$

\section{Definitions}

Based on modifications of those suggested by Friedman et al. ${ }^{20}$ a positive blood culture was classified as communityacquired if collected within $48 \mathrm{~h}$ of admission in a patient who had not been admitted to hospital in the previous 3 months, and as hospital-acquired if collected after $48 \mathrm{~h}$ of admission or if the patient had been admitted to hospital in the previous 3 months. ${ }^{20}$

\section{Statistical analysis}

Data were analysed using Stata version 14.2 (Stata Corp, College Station, TX) and Microsoft Excel. Summary statistics were used to describe clinical, laboratory and epidemiological characteristics. Categorical variables were compared between groups using the chi-square test.

Significant differences between groups were defined as $p \leq$ 0.05. Receiver operating characteristic curves were generated to determine the optimum real-time PCR CT cutoff value for positivity. Diagnostic accuracy (sensitivity, specificity, positive predictive values and negative predictive values with $95 \% \mathrm{CI}$ ) was calculated using the DIAGTEST Stata module.

\section{Ethical considerations}

The study was approved by the Human Research Ethics Committee at the University of Cape Town (HREC REF Number 729/2015).

\section{Results}

A total of 212 signal-positive blood cultures with morphology meeting the specified inclusion criteria from 193 patients were selected. Multiple samples from a single patient were included if collected on different days or times or from different lumens of a central venous catheter.

\section{Culture results}

Sub-culture from blood culture broth was positive for 195/212 (92\%) samples, where S. pneumoniae was isolated from 55/212 (26\%) samples (Figure 1a). Of these 55 samples, three $(5 \%)$ were mixed cultures with Listeria monocytogenes, Klebsiella pneumoniae or a coagulase negative staphylococcus (CoNS). Other gram-positive organisms were cultured in 140/212 (66\%) samples, whilst the remaining $17 / 212$ samples $(8 \%)$ were sub-culture negative.

We stratified samples according to culture results into the S. pneumoniae (Spn) group, the non-S. pneumoniae (NSpn) (other gram-positive organisms) group and the no growth (NG) on sub-culture group. Characteristics of the groups are summarised in Table 2-A1.

Visual autolysis occurred in 33\% of all samples collected (Figure 1c). The proportion of visually autolysed samples differed significantly between groups, with $94 \%(16 / 17)$ of samples in the NG group, 56\% (31/55) of samples in the Spn group and $16 \%(22 / 140)$ of samples in the NSpn exhibiting visible autolysis $(p \leq 0.001)$.

\section{Performance of polymerase chain reaction and antigen tests}

Overall, we detected lyt $A$ in 71/212 (33\%) samples using PCR, and ICT was positive in $89 / 212$ (42\%) samples (Figure 1a). There were 193/212 (91\%) samples which had concordant culture, PCR and antigen test results.

The results of the 19/212 (9\%) discordant samples are summarised in Table 1.

\section{Clinical, laboratory and epidemiological characteristics}

Respiratory illness and meningitis predominated in the Spn (75\%) and NG (65\%) groups, whilst other illnesses predominated in the NSpn (61\%) group and this was significant $(p \leq 0.001)$.

Gram-positive cocci in pairs were significant in the both of the Spn (69\%) and NG groups (88\%), whereas the proportion with this morphology in the NSpn group was only 19\% $(p<0.001)$ (Figure 1d).

Most samples in the Spn (78\%) and NG (88\%) groups were submitted from primary/secondary level care hospitals and most samples from the Spn (67\%) and NG groups (82\%) fulfilled our definition for community-acquired infection. Fifty-three per cent of samples in the NSpn group were also sent from primary/secondary level care hospitals, and 63\% of samples in the NSpn group fulfilled our definition for (HAI) $(p \leq 0.001)$ (Figure 1f). One sample could not be assigned to community or HAI categories because of missing data. 

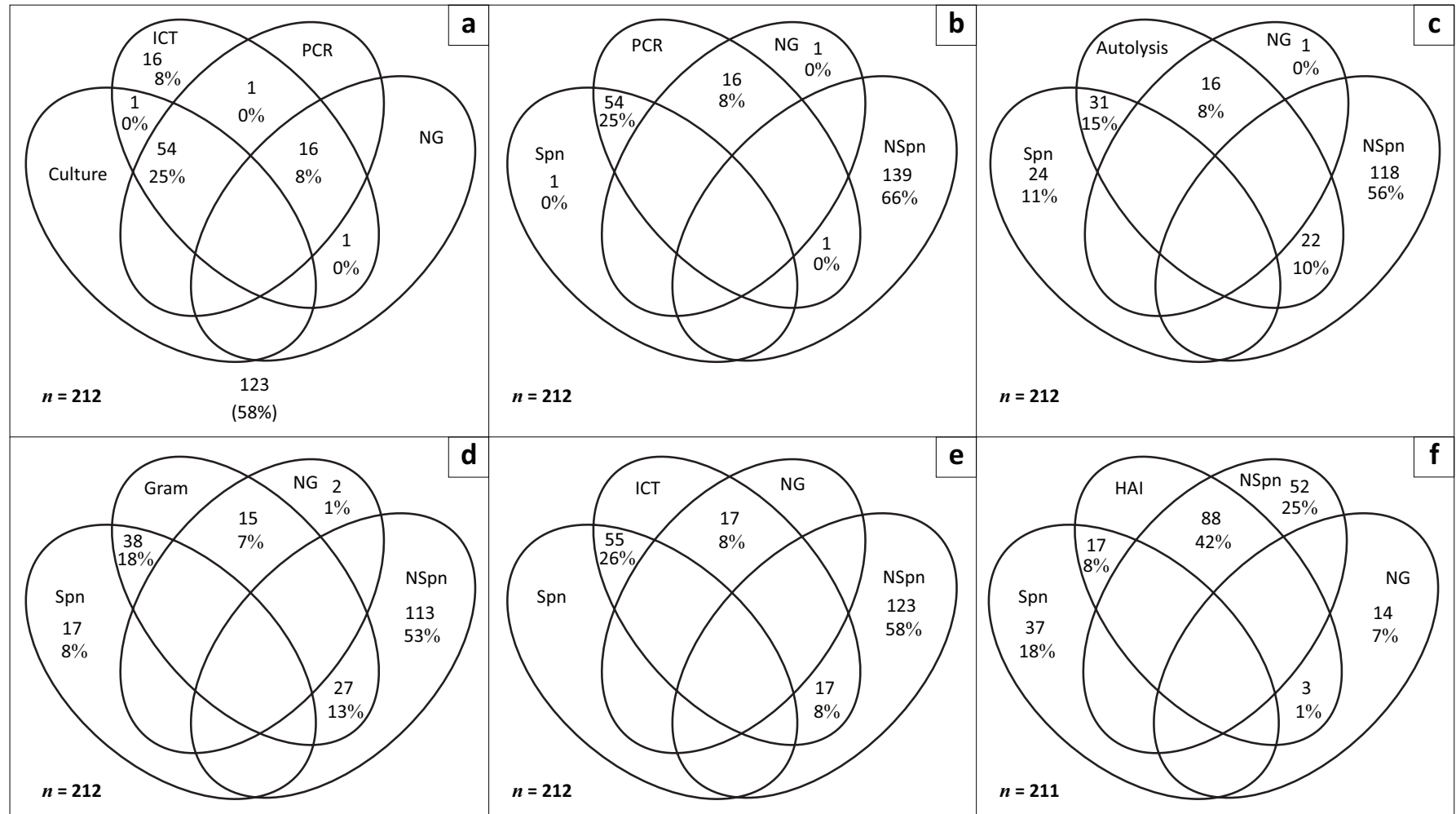

ICT, immunochromatographic test; PCR, polymerase chain reaction; HAI, hospital-acquired infection; Spn, S. pneumoniae; NSpn, non-S. pneumoniae; NG, no growth

FIGURE 1: Venn diagrams representing numbers and proportions of specimens tested with (a) culture positivity versus immunochromatographic test and PCR positivity; (b) PCR positivity versus culture stratified into Streptococcus pneumoniae, non-Streptococcus pneumoniae and NG groups; (c) autolysis versus culture positivity stratified into Streptococcus pneumoniae, non-Streptococcus pneumoniae and no growth groups; (d) Gram stain with gram-positive cocci in pairs ('Gram') versus culture positivity stratified into Streptococcus pneumoniae, non-Streptococcus pneumoniae and no growth groups; (e) immunochromatographic test versus culture stratified into Streptococcus pneumoniae, non-Streptococcus pneumoniae and no growth groups; and (f) samples that met the study definition for hospital-acquired infection versus culture positivity stratified into Streptococcus pneumoniae, non-Streptococcus pneumoniae and no growth groups.

TABLE 1: Discordant results between the antigen test (immunochromatographic test), polymerase chain reaction and culture.

\begin{tabular}{|c|c|c|c|c|}
\hline Number & Culture result & Antigen (ICT) & PCR & CT value: \\
\hline \multicolumn{5}{|c|}{ Culture and antigen positive for S. pneumoniae but PCR negative } \\
\hline 1 & Streptococcus pneumoniae + coagulase negative staphylococcus & Positive & Negative & 26.00 \\
\hline \multicolumn{5}{|c|}{ Culture negative for S. pneumoniae but antigen and PCR positive for S. pneumoniae } \\
\hline 2 & Coagulase negative staphylococcus & Positive & Positive & 13.76 \\
\hline \multicolumn{5}{|c|}{ Culture and PCR negative for $S$. pneumoniae but antigen positive } \\
\hline 3 & Enterococcus faecium + coagulase negative staphylococcus & Weak positive & Negative & - \\
\hline 4 & Streptococcus agalactiae & Weak positive & Negative & - \\
\hline 5 & Streptococcus anginosus & Weak positive & Negative & - \\
\hline 6 & Enterococcus faecalis + coagulase negative staphylococcus & Weak positive & Negative & - \\
\hline 7 & Viridans streptococcus & Positive & Negative & - \\
\hline 8 & Viridans streptococcus † & Positive & Negative & 25.04 \\
\hline 9 & Streptococcus mitis † & Positive & Negative & 26.49 \\
\hline 10 & Streptococcus mitis † & Positive & Negative & 26.32 \\
\hline 11 & Streptococcus mitis & Positive & Negative & 25.69 \\
\hline 13 & Streptococcus mitis + coagulase negative staphylococcus & Positive & Negative & - \\
\hline 14 & Streptococcus alactolyticus & Positive & Negative & - \\
\hline 15 & Coagulase negative staphylococcus & Positive & Negative & - \\
\hline 16 & Skin flora & Positive & Negative & 29.22 \\
\hline 17 & Skin flora & Positive & Negative & - \\
\hline 18 & Skin flora & Positive & Negative & - \\
\hline \multicolumn{5}{|c|}{ No growth on culture and PCR negative but antigen positive } \\
\hline 19 & No growth & Positive & Negative & - \\
\hline
\end{tabular}

ICT, immunochromatographic test; $\mathrm{PCR}$, polymerase chain reaction; $\mathrm{CT}$, cycle threshold; S. pneumoniae, Streptococcus pneumoniae.

$\dagger$, Isolates from the same patient; $\ddagger$, A cycle threshold cut-off value of $\leq 22$ was used to determine PCR positivity; $\S$, Skin flora were mixed viridans streptococci \pm coagulase negative staphylococcus. 


\section{Diagnostic accuracy}

The sensitivity of ICT compared with culture, PCR and the composite of culture or PCR was $100 \%$. Specificity was $88 \%$, $87 \%$ and $88 \%$, respectively. Positive predictive value was $76 \%$, $80 \%$ and $81 \%$, respectively, whilst the negative predictive value was $100 \%$. Specificity and positive predictive value against each of these reference standards increased to $93 \%-96 \%$ and $96 \%$ $98 \%$ when applied to subsets of samples with gram-positive cocci in pairs on Gram stain, or subsets of samples with a clinical history suggestive of respiratory illness or meningitis. The immunochromatographic test was positive for all 72 samples that were positive for $S$. pneumoniae using our composite reference, even though only 53 of these samples had grampositive cocci in pairs on Gram stain. Analysis after exclusion of duplicate samples did not alter the results significantly.

The sensitivity of classical pneumococcal Gram stain with gram-positive cocci in pairs in our study was $69 \%, 75 \%$ and $74 \%$ when compared with culture, PCR and the composite reference, and specificity was $81 \%$ for all three. The positive predictive values were $58 \%, 66 \%$ and $66 \%$. The negative predictive values were $87 \%, 86 \%$ and $86 \%$. The sensitivity decreased and specificity was unchanged when applied to the subset with a history compatible with respiratory illness or meningitis. Whilst positive predictive values increased when applied only to this subset, this was still below $90 \%$ and also resulted in a decrease in the negative predictive value of $\leq 60 \%$. Analysis did not change significantly after duplicate samples were excluded.

\section{Discussion}

Rapid diagnosis of IPD is challenging and current methods are limited by various factors. We aimed to demonstrate the utility of a selected ICT to rapidly identify S. pneumoniae on broth from selected signal-positive blood cultures.

The immunochromatographic tests were positive in all samples in the Spn (sub-culture positive for S. pneumoniae; $n=55$ ) group, giving a sensitivity of $100 \%$. Only one sample (Case 1) in this group was ICT positive but PCR negative, with a CT value of 26. Culture results of this sample indicated mixed growth of S. pneumoniae and a CoNS, and the elevated CT value was likely because of growth competition with the CoNS for nutrients in the blood culture broth.

The immunochromatographic tests were positive in all samples in the NG (no growth on culture; $n=17$ ) group. All but one sample (1/17) in the NG group (Case 19) was PCR positive, suggesting a probable false-positive ICT result. Retesting of Case 19 with a different PCR was negative (data not shown) and the patient was subsequently diagnosed with enterovirus meningitis on viral PCR. The similarities between ICT results obtained for the Spn and NG groups support the assumption that samples in the NG group most likely represented autolysed, non-viable $S$. pneumoniae and that the addition of a culture-independent assay for these types of results may assist diagnosis. 
The NSpn group (sub-culture culture positive for organisms other than S. pneumoniae; $n=140$ ) had 17 samples which were ICT positive; of these, only Case 2 was also PCR positive (CT = 13.76). Gram-positive cocci were observed for this sample, there was no visible autolysis and culture yielded a CoNS. It is unlikely that both ICT and PCR produced false-positive results, especially with the low CT value obtained. A likely explanation is that S. pneumoniae was either non-viable or outcompeted by the CoNS on culture. Of the remaining ICT positive samples, 5/16 produced a detectable signal on PCR, above the determined cut-off, with CT values ranging from 25 to 29 (cases 8-11 and 16). It is possible that non-viable $S$. pneumoniae was present or outcompeted by growth of other organisms including viridans streptococci in these samples. Alternatively, the signal detected could have been because of the cross-reactivity of the PCR with other gram-positive organisms, which has mostly been described in the presence of other streptococcal species owing to their genetic relatedness. ${ }^{21}$

Our reported ICT sensitivity (100\%) was similar to that reported by Altun and colleagues (100\%) and Moisi and colleagues $(87 \%-100 \%)$. However, our reported specificity $(87 \%-88 \%)$ was higher than that reported by Altun and colleagues (64\%) and lower than that reported by Moisi and colleagues $(96 \%-100 \%)$. Altun and colleagues compared ICT with culture, directly on signal-positive blood cultures with gram-positive cocci in pairs or chains. However, the distribution of gram-positive cocci in pairs versus chains was not described and no confirmatory molecular testing was performed; therefore, the presence of non-viable $S$. pneumoniae in samples could not be excluded. ${ }^{13}$ Moisi and colleagues performed ICT and lytA PCR directly on any signal-positive blood cultures in Mali and Thailand, irrespective of Gram stain results, and ICT was only compared with culture and not PCR as a reference method. The inclusion of any signal-positive sample, irrespective of Gram stain finding, may account for the higher value reported for specificity. ${ }^{12}$

Classic Gram stain morphology of S. pneumoniae (grampositive cocci in pairs) performed poorly in the diagnosis of IPD, with limited sensitivity $(69 \%-75 \%)$, specificity $(81 \%)$, positive predictive $(58 \%-66 \%)$ and negative predictive values $(86 \%-87 \%)$. Even though the positive predictive value improved to $84 \%-88 \%$ when applied to the subset of samples with a history compatible with respiratory illness or meningitis, this resulted in a decrease in the negative predictive value $(57 \%-60 \%)$ and sensitivity $(66 \%-71 \%)$, limiting its utility as a standalone diagnostic tool for IPD. Specificity (81\%) remained unchanged when applied to this subset.

An important limiting factor when assessing diagnostic tools for S. pneumoniae diagnosis is the lack of a reliable gold standard, with poor sensitivity for culture. We included lyt $A$ PCR as a reference standard, but used a stringent CT cut-off value to compensate for its recognised limited specificity. The CT cut-off value of $\leq 22$ to determine positivity was, therefore, considerably lower than the conventional diagnostic PCR CT cut-off value (CT 37), but because the potential target organism was already enriched in the blood culture broth, and the extracted DNA diluted 1:10 prior to PCR, a lower CT cut-off value was deemed acceptable. This experimentally determined cut-off, however, resulted in one false-negative PCR result when compared with culture, and five samples, which were ICT positive and produced a signal on PCR above the determined cut-off, but were Spn culturenegative. Experimentally determined cut-off values should be used with caution and interpreted in conjunction with other laboratory findings.

We reported faint ICT results as positive, according to the manufacturer's instructions. We noted four such weak positive results, all of which cultured other gram-positive organisms, and were PCR negative (no signal detected). Whilst the numbers were small, we recommend reporting weak positive results as negative or indeterminate.

We observed significant differences in basic clinical and epidemiological characteristics and Gram stain morphology between the Spn, NG and NSpn groups, with the majority of isolates from the Spn and NG groups exhibiting gram-positive cocci in pairs $(69 \%$ and $88 \%)$, autolysis (56\% and 94\%) and occurring in patients with primarily community-acquired infection (67\% and $82 \%$ ), which were predominantly respiratory illness and meningitis (75\% and 65\%).

In contrast, isolates from the NSpn group exhibited grampositive cocci in pairs in only $19 \%$ of samples, were rarely visibly autolysed (16\%), were predominantly isolated from patients who met our definition for HAI $(63 \%)$ and most (61\%) presented with illnesses other than respiratory illness or meningitis.

There were insufficient data regarding prior antibiotic exposure to permit analysis for the different groups.

\section{Conclusion}

We found that ICT had a high sensitivity and would therefore be a useful rule-out test for S. pneumoniae bacteraemia. A positive result could provide a rapid presumptive diagnosis of S. pneumoniae bacteraemia, especially where Gram stain morphology and clinical history are available to offset limitations in specificity. We conclude that the BinaxNOW pneumococcal antigen test is a useful adjunct for the diagnosis of pneumococcal bacteraemia. The added cost of the BinaxNOW pneumococcal antigen test from Alere was modest compared with DNA extraction and PCR. In our setting, where high-level penicillin resistance is relatively rare, the early identification of S. pneumoniae permits rapid de-escalation to penicillin in the appropriate non-meningitis clinical setting, with the potential to impact patient care and improve antibiotic stewardship. The immunochromatographic test is potentially a suitable alternative to PCR for the detection of $S$. pneumoniae in culture-negative specimens, but because of small numbers of culture-negative samples in our study, this should be further validated. 


\section{Acknowledgements}

The authors would like to acknowledge the staff of the National Health Laboratory Service microbiology laboratory at Groote Schuur Hospital, Cape Town and staff at the Division of Medical Microbiology, University of Cape Town for their support.

\section{Competing interests}

The authors declare that they have no financial or personal relationships that may have inappropriately influenced them in writing this research article.

\section{Authors' contributions}

H.T., C.M. and C.B. designed the study. H.T. and C.M. performed the lab tests. H.T. and C.M.C. analysed and interpreted the data. H.T. wrote the manuscript in consultation with and support from C.M., C.B. and C.M.C.

\section{Funding information}

This work was supported by funding from the NHLS Research Trust.

\section{Data availability}

The data that support the findings of this study are available from the corresponding author, C.M., upon reasonable request.

\section{Disclaimer}

The views and opinions expressed in this article are those of the authors and do not necessarily reflect the official policy or position of any affiliated agency of the authors.

\section{References}

1. GERMS Annual Report - National Institute for Communicable Diseases (South Africa) [homepage on the Internet]. 2017 [cited n.d.]. Available from: http://www. nicd.ac.za/index.php/publications/germs-annual-reports/

2. Von Gottberg A, De Gouveia L, Tempia S, et al. Effects of vaccination on invasive pneumococcal disease in South Africa. N Engl J Med. 2014;371(20):1889-1899. https://doi.org/10.1056/NEJMoa1401914

3. Wahl B, O'Brien KL, Greenbaum A, et al. Burden of Streptococcus pneumoniae and Haemophilus influenzae type $b$ disease in children in the era of conjugate vaccines: Global, regional, and national estimates for 2000-2015. Lancet Glob Health. 2018;6(7):e744-e757. https://doi.org/10.1016/S2214-109X(18)30247-X
4. Bandettini R, Melioli G. Laboratory diagnosis of Streptococcus pneumoniae infections: Past and future. J Prev Med Hyg. 2012;53(2):85-88.

5. Baggett HC, Rhodes J, Dejsirilert S, et al. Pneumococcal antigen testing of blood culture broth to enhance the detection of Streptococcus pneumoniae bacteremia. Eur J Clin Microbiol Infect Dis. 2012:31(5):753-756. https://doi.org/10.1007/ s10096-011-1370-3

6. Petti CA, Woods CW, Reller LB. Streptococcus pneumoniae antigen test using positive blood culture bottles as an alternative method to diagnose pneumococcal bacteremia. J Clin Microbiol. 2005;43(5):2510-2512. https://doi.org/10.1128/ JCM.43.5.2510-2512.2005

7. Casado Flores J, Nieto Moro $M$, Berron $S$, Jimenez $R$, Casal J. Usefulness of pneumococcal antigen detection in pleural effusion for the rapid diagnosis of infection by Streptococcus pneumoniae. Eur J Pediatr. 2010;169(5):581-584. infection by Streptococcus pneumoniae. Eur
https://doi.org/10.1007/s00431-009-1077-y

8. Samra Z, Shmuely H, Nahum E, Paghis D, Ben-Ari J. Use of the NOW Streptococcus pneumoniae urinary antigen test in cerebrospinal fluid for rapid diagnosis of pneumoniae urinary antigen test in cerebrospinal fluid for rapid diagnosis of
pneumococcal meningitis. Diagn Microbiol Infect Dis. 2003;45(4):237-240. pneumococcal meningitis. Diagn Microbiol In
https://doi.org/10.1016/S0732-8893(02)00548-5

9. Varghese $R$, Jayaraman $R$, Veeraraghavan $B$. Current challenges in the accurate identification of Streptococcus pneumoniae and its serogroups/serotypes in the vaccine era. J Microbiol Methods. 2017;141:48-54. https://doi.org/10.1016/j. mimet.2017.07.015

10. Sinclair A, Xie X, Teltscher M, Dendukuri N. Systematic review and meta-analysis of a urine-based pneumococcal antigen test for diagnosis of community-acquired pneumonia caused by Streptococcus pneumoniae. J Clin Microbiol. 2013;51(7):2303-2310. https://doi.org/10.1128/JCM.00137-13

11. Marcos MA, Martinez E, Almela M, Mensa J, Jimenez de Anta MT. New rapid antigen test for diagnosis of pneumococcal meningitis. Lancet. 2001;357(9267):1499-1500. https://doi.org/10.1016/S0140-6736(00)04658-4

12. Moisi JC, Moore M, Carvalho M da G, et al. Enhanced diagnosis of pneumococcal bacteremia using antigen- and molecular-based tools on blood specimens in Mali and Thailand: A prospective surveillance study. Am J Trop Med Hyg. 2016;94(2):267-275. https://doi.org/10.4269/ajtmh.15-0431

13. Altun $\mathrm{O}$, Athlin S, Almuhayawi M, Stralin K, Ozenci V. Rapid identification of Streptococcus pneumoniae in blood cultures by using the ImmuLex, Slidex and Wellcogen latex agglutination tests and the BinaxNOW antigen test. Eur J Clin Microbio Infect Dis. 2016;35(4):579-585. https://doi.org/10.1007/s10096-015-2573-9

14. Isenberg HD. Clinical microbiology procedures handbook. 2nd ed. Update. Washington DC: American Society of Microbiology; 2007.

15. Alere. BinaxNOW'M S. pneumoniae antigen card package insert. 2020 [cited n.d.] Available from: https://www.globalpointofcare.abbott/en/product-details/binaxnowstreptococcus-pneumoniae-ous.html.

16. Carvalho M da G, Tondella ML, McCaustland K, et al. Evaluation and improvement of real-time PCR assays targeting lytA, ply, and psaA genes for detection of pneumococca DNA. J Clin Microbiol. 2007;45(8):2460-2466. https://doi.org/10.1128/JCM.02498-06

17. Lin N. Sample size for sensitivity \& specificity studies [homepage on the Internet]. Universiti Sains Malaysia. 2004 [cited n.d.]. Available from: https:// www.researchgate.net/profile/Stephen_Reynolds13/post/How-can-Idetermine-an-adequate-sample-size-for-a-clinical-trial-when-I-use-reagentswith-known-sensitivity-and-specifity/attachment/5ecd103ff06ddf00011a001b/ AS\%3A895424911122432\%401590497343040/download/ Sample+Size+Calculator.xls

18. Jones SR, Carley S, Harrison M. An introduction to power and sample size estimation Emerg Med J. 2003;20(5):453-458. https://doi.org/10.1136/emj.20.5.453

19. Buderer NM. Statistical methodology: I. Incorporating the prevalence of disease into the sample size calculation for sensitivity and specificity. Acad Emerg Med. 1996;3(9):895-900. https://doi.org/10.1111/j.1553-2712.1996.tb03538.x

20. Friedman ND, Kaye KS, Stout JE, et al. Health care-associated bloodstream infections in adults: A reason to change the accepted definition of communityacquired infections. Ann Intern Med. 2002;137(10):791-797. https://doi. org/10.7326/0003-4819-137-10-200211190-00007

21. Donati C, Hiller NL, Tettelin H, et al. Structure and dynamics of the pan-genome of Streptococcus pneumoniae and closely related species. Genome Biol. 2010;11(10):R107. https://doi.org/10.1186/gb-2010-11-10-r107 


\section{Appendix 1}

TABLE 1-A1: Primer and probe sequences used for lytA PCR assay.

\begin{tabular}{ll}
\hline Primer or probe & Sequence \\
\hline SPN Forward primer & $5^{\prime}$ - TCG TGC GTT TTA ATT CCA GCT -3' \\
SPN Reverse primer & $5^{\prime}$ - ACG CAA TCT AGC AGA TGA AGC A -3' \\
SPN Probe & $5^{\prime}-/ 56-F A M / C T C$ CCT GTA /ZEN/TCA AGC GTT \\
& TTC GGC A/3IABkFQ/ -3' \\
\hline
\end{tabular}

TABLE 2-A1: Clinical, laboratory and epidemiological characteristics of samples grouped according to culture.

\begin{tabular}{|c|c|c|c|c|c|c|c|c|c|}
\hline \multirow[t]{3}{*}{ Variable } & \multicolumn{6}{|c|}{ Group } & \multicolumn{3}{|c|}{ Statistical comparison ( $p$-value) } \\
\hline & \multicolumn{2}{|c|}{ Spn $(N=55)$} & \multicolumn{2}{|c|}{ NSpn $(N=140)$} & \multicolumn{2}{|c|}{ NG $(N=17)$} & \multirow[t]{2}{*}{ Spn vs NSpn vs NG } & \multirow[t]{2}{*}{ Spn vs. NSpn } & \multirow[t]{2}{*}{ Spn vs. NG } \\
\hline & $N$ & $\%$ & $N$ & $\%$ & $N$ & $\%$ & & & \\
\hline Clinical & - & - & - & - & - & - & $<0.001$ & $<0.001$ & 0.884 \\
\hline Respiratory illness & 34 & 62 & 22 & 16 & 9 & 53 & - & - & - \\
\hline Meningitis & 7 & 13 & 4 & 3 & 2 & 12 & - & - & - \\
\hline Other & 2 & 4 & 86 & 61 & 1 & 6 & - & - & - \\
\hline No data & 12 & 22 & 28 & 20 & 5 & 9 & - & - & - \\
\hline Autolysis $\dagger$ & - & - & - & - & - & - & $<0.001$ & $<0.001$ & 0.004 \\
\hline Yes & 31 & 56 & 22 & 16 & 16 & 94 & - & - & - \\
\hline No & 24 & 44 & 118 & 84 & 1 & 6 & - & - & - \\
\hline Gram stain & - & - & - & - & - & - & $<0.001$ & $<0.001$ & 0.112 \\
\hline Pairs & 38 & 69 & 27 & 19 & 15 & 88 & - & - & - \\
\hline Chains & 16 & 29 & 63 & 45 & 1 & 6 & - & - & - \\
\hline Cocci & 1 & 2 & 50 & 36 & 1 & 6 & - & - & - \\
\hline Tertiary level care hospital & 12 & 22 & 66 & 47 & 2 & 12 & - & - & - \\
\hline $\begin{array}{l}\text { Primary or secondary level care } \\
\text { hospitals }\end{array}$ & 43 & 78 & 74 & 53 & 15 & 88 & - & - & - \\
\hline $\begin{array}{l}\text { Community versus nosocomial } \\
\text { infection: }\end{array}$ & - & - & - & - & - & - & $<0.001$ & $<0.001$ & 0.461 \\
\hline Community & 37 & 67 & 52 & 37 & 14 & 82 & - & - & - \\
\hline Nosocomial & 17 & 31 & 88 & 63 & 3 & 18 & - & - & - \\
\hline No data & 1 & 2 & 0 & 0 & 0 & 0 & - & - & - \\
\hline
\end{tabular}

Spn, Growth of S. pneumoniae on culture; NSpn, Growth of an organism other than S. pneumoniae on culture; NG, No growth of any organism on culture.

$\dagger$, Autolysis was determined by the subjective presence of brown discoloration of the blood culture broth; $\$$, Community versus nosocomial infection was determined as per the study definition. 


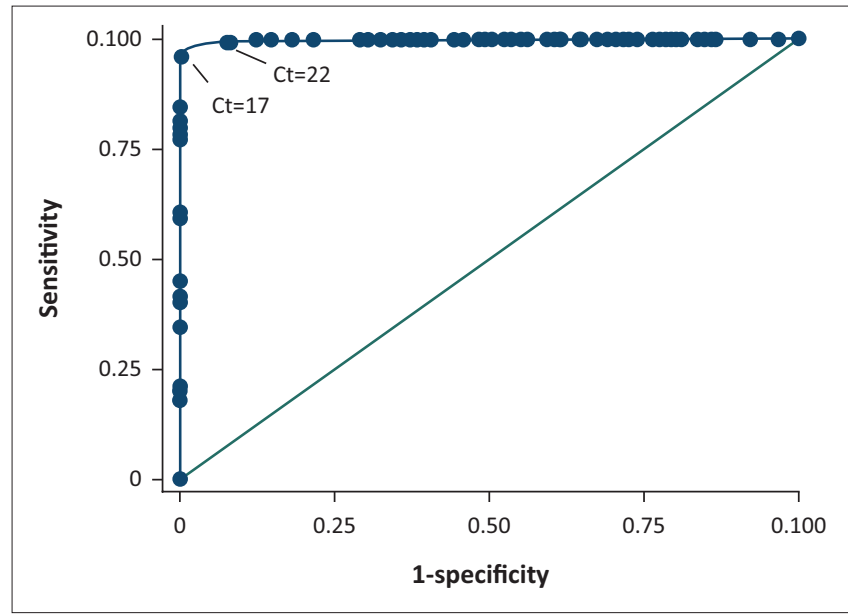

FIGURE 1-A1: Receiver operating characteristic (ROC) curve analysis was used to determine the optimal CT cut-off value for determining a positive PCR result. Samples culturing S. pneumoniae were regarded as true positives, and samples culturing organisms other than S. pneumoniae were regarded as true negatives for this analysis. ROC curve analysis suggested that the optimal CT cut-off value for determining a positive PCR result was between 17 and 22 (AUC 0.99), and the upper value of 22 was selected.

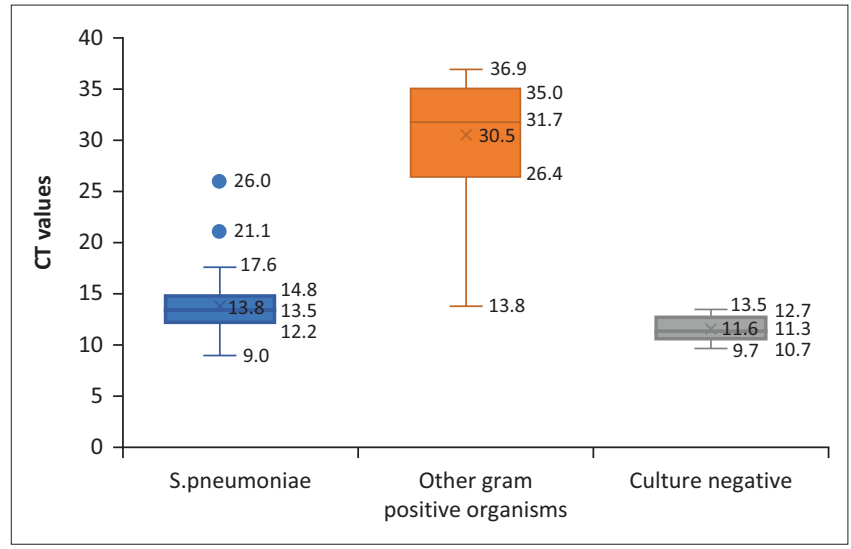

$\mathrm{CT}$, cycle threshold.

FIGURE 2-A1: Distribution of PCR cycle threshold (CT) values using box and whisker diagrams, in signal-positive blood culture samples that have been grouped based on culture results. Whiskers represent the minimum and maximum values of the CT-values and boxes represents the interquartile range. ' $\mathrm{X}$ ' represents the mean of CT-values. For the S. pneumoniae group, there were 2 CT-values that were outliers (excluded from box and whisker diagram) and these are represented by dots in the figure. 